\title{
Piceatannol induced apoptosis through up-regulation of microRNA-181a in melanoma cells
}

\author{
Maotao Du* ${ }^{*}$ Zhong Zhang ${ }^{2}$ and Tao Gao ${ }^{2}$
}

\begin{abstract}
Background: Melanoma took top position among the lethal cancers and, despite there have been some great attempts made to increase the natural life of patients with metastatic disease, long-lasting and complete remissions are few. Piceatannol, owns the similar function as resveratrol, has been defined as an anti-cancer agent playing important role in inhibition of proliferation, migration and metastasis in various cancer. Thus, we aim to investigate the anti-cancer effect and mechanisms of piceatannol in melanoma cells.

Methods: Melanoma cell lines WM266-4 and A2058 were treated either with or without piceatannol. Cell viability and cell apoptosis were assessed by using MTT and Annexin V/PI assay, respectively. Cells were transfected with specific miRNA using Lipfectamine 2000. miRNA bingding ability to 3'-UTR region within specific gene was assed by firefly luciferase analysis. Gene and protein expression was eveluated by qRT-PCR and western blot analysis, respectively.

Results: Our study showed that piceatannol inhibited WM266-4 and A2058 cells growth and induced apoptosis. Totally, 16 differentially expressed miRNAs were screened out including 8 up-regulated and 8 down-regulated miRNAs. Expression level of miR-181a is significantly higher in piceatannol-treated cells than normal control and is lower in melanoma cancer tissues than its adjacent normal tissues. Bcl-2 is a target gene of miR-181a. Moreover, silencing of miR-181a reverses the decrease of cell viability induced by piceatannol in WM266-4 and A2058 cells. Taken together, present study uncovered the ability of piceatannol to repress melanoma cell growth and clarified the contribution of miR-181a in the anticancer role of piceatannol.
\end{abstract}

Conclusion: The present study proposes that piceatannol can be taken into account to be a hopeful anticancer agent for melanoma.

Keywords: Piceatannol, Apoptosis, microRNA-181-a, Melanoma cells

\section{Background}

Melanoma is aggressive skin cancer and its incidence continues to increase globally $[1,2]$. It is the fifth and sixth most common cancer in males and females, respectively [3], and is one of the most common cancers among adolescents and young adults [4]. It is known that surgical resection is a promising and curative intervention when melanoma is diagnosed at early stage, but many patients

\footnotetext{
*Correspondence: motaodu@163.com

1 Department of Dermatology, The Second Affiliated Hospital

of Chongqing Medical University, 76 Linjiang Road, Yuzhong District,

Chongqing 400010, China

Full list of author information is available at the end of the article
}

show up with unresectable cancer at later stages which is usually treated with surgical operation, commonly together with adjuvant chemotherapy [5]. By reason of its complex etiology addition to drug/chemo resistance and high metastatic potential, poor prognosis often present in patients who are diagnosed with advanced melanoma, remaining therapeutically unsatisfactory for many years [6]. For cancer chemo prevention or treatment, the discovery and development of novel medicament with low toxicity, high efficiency and excellent potential is an important part of cancer therapies. Thus, it is needed to find effective bio-compound by studying its molecular mechanism at the basis of melanoma pathogenesis in 
order to provide potential targets for its diagnosis and treatment.

Piceatannol is a strong antioxidant and owns anticancer and chemo preventive abilities. Despite the pharmacological actions of piceatannol, especially its antioxidant, antitumor, and anti-inflammatory activities, including bioavailability and toxicity in humans are well documented, depending on the cell type piceatannol may either stimulate [7, 8] or inhibit [7] apoptosis. Although it was reported that the evaluation of anti-melanoma effect of piceatannol on human melanoma cells SK-Mel-28 [9], the results limited with supporting metabolic data and therapeutic effects and underlying mechanism of piceatannol on human melanoma remains unknown. Thereby, these limitations are attempted to be replenished in our study.

It is the fact that microRNAs (miRs) are a class of small non-coding RNA that can specifically binding to the $3^{\prime}$-untranslational region of their target mRNAs and in this way cause mRNA degradation or inhibition of protein translation $[10,11]$. Altered miRNA expression has been widely reported in cancer, it has been reported that high levels of oncogenic miRNAs downregulate tumor suppressor genes, and conversely, decreased tumor suppressor miRNAs responsible for elevated oncogene expression [12]. By means of regulating the expression of their target genes, miRNAs affect diverse of biological processes, such as cell proliferation, survival, differentiation, cell cycle progression and apoptosis [13]. In addition to their prognostic or diagnostic utility as biomarkers, miRNAs have appeared as novel biological tool for cancer treatment [14-16]. It is complex to escape from apoptosis for cancer cells. Highly expressed levels of anti-apoptotic protein, B cell lymphoma $2(\mathrm{Bcl}-2)$ is known to be one of the major causal factors in tumorigenesis. In the apoptotic process of eukaryotic cells, Bcl-2 plays an crucial role contributing survival in inhibition of cell death [17]. Highly expressed level of Bcl-2 has been reported in various cancers including melanoma [18], and has been broadly linked to its anti-apoptotic activity in melanoma cells, silencing by miRNAs [19].

In present study, we attempt to investigate the anticancer mechanisms of piceatannol in the highly aggressive human melanoma cell line, WM266-4 and A2058, and elucidate the inhibitory effect of piceatannol on melanoma cells through attenuating miRNA-mediated Bcl-2.

\section{Methods}

\section{Materials and samples}

Piceatannol with purity > 99\% was obtained from Chengdu Must Bio-tech, and $100 \mathrm{mM}$ stock solution was prepared by dissolving the piceatannol in ethanol and stored at $-20{ }^{\circ} \mathrm{C}$ in the dark. MTT based Cell Counting Kit-8 (CCK-8) (Beyotime, Haimen, China), 10\% fetal bovine serum (Gibco, UK), antibodies of Bcl-2, Bax, caspase- 3 and $\beta$-actin were provided from Abcam (Cambridge, MA).

Tissue specimens were obtained from patients who are underwent melanoma surgical resection in the Second Affiliated Hospital of Chongqing Medical University and adjacent tissues, which is classified as control group, were acquired from resected neighboring area of melanoma. We surgically procured tumor samples from patients with primary cutaneous melanoma. Upon surgical removal of the primary melanoma, a single surgical oncologist (A.I.R.) used a scalpel to macrodissect and procured a portion of the remaining primary tumor. We've collected 10 paired tissues, namely 10 normal tissues and 10 tumor tissues, in which the level of miR181a was measured. No patients underwent cutaneous radiotherapy or chemotherapy before. This study was approved by ethics review board of Chongqing Medical University and all the patients provided written informed consent.

\section{Cell line, culture and piceatannol treatment}

Melanoma cells WM266-4 and A2058 were provided from Wuhan cell bank of Procell. Cells were cultured in DMEM contains $10 \%$ fetal bovine serum, $100 \mu \mathrm{g} / \mathrm{ml}$ of penicillin and streptomycin, and incubated in a humidified condition of $95 \%$ air and $5 \% \mathrm{CO}_{2}$ at $37{ }^{\circ} \mathrm{C}$. After 24 and $48 \mathrm{~h}$ of culturing, the cells treated with piceatannol were collected for following measurement.

\section{MTT cell viability assay}

The 96-hole culture plates was used to seed WM266-4 and A2058 cells with $1 \times 10$ [4] cells/well, and the plate was placed in incubation box at $37{ }^{\circ} \mathrm{C}$ with $5 \% \mathrm{CO}_{2}$. The cells were treated with different concentrations of piceatannol and after 24-h culture, MTT assay (Gibco) was performed to determine the cell apoptosis. The culture media were placed and $20 \mu \mathrm{l} \mathrm{MTT}$ assay $(5 \mathrm{mg} /$ $\mathrm{ml}$ ) was added to each well prior to $4 \mathrm{~h}$ incubation. $150 \mu \mathrm{l}$ DMSO were added to dissolve the formazan crystals. After $10 \mathrm{~min}$ oscillation, the optical density (OD) values were measured with a microplate reader (Beckmann Coulters) at $490 \mathrm{~nm}$. Cell viability was presented as a percent of MTT reduction in the treated cells versus the controls (cells incubated in serumfree DMEM without extracts). The relative MTT level (\%) was calculated as $[\mathrm{A}] /[\mathrm{B}] \times 100$, where $[\mathrm{A}]$ is the absorbance of the test sample and [B] is the absorbance of control sample containing the untreated cells. Decreased relative MTT level indicates decreased cell viability. 


\section{Cell apoptosis detection by flow cytometry}

In brief, WM266-4 and A2058 cells were harvested and washed with PBS after treatment. The density of cells was adjusted to $3 \times 10$ [5], then added $400 \mu \mathrm{l}$ binding buffer, $5 \mu$ l Annexin V (Gibco), $10 \mu \mathrm{l}$ PI (Bio-Science, Shanghai, China), and placed the plate at room temperature for $15 \mathrm{~min}$ in the dark. Flow cytometry (LSRII, BD Biosciences) was used to analyze all the samples. Statistical analysis of flow cytometry-obtained apoptosis (\%) in both WM266-4 and A2058 cell lines was performed using t test to generate $P$ value in order to compute the difference between treated and untreated scores. Statistical analysis between untreated and control and between treated and control was also presented.

\section{Western blot analysis}

The total proteins were extracted from WM266-4 and A2058 cells. Cells were lysed in RIPA buffer (Beyotime Institute of Biotechnology, Haimen, China) for $40 \mathrm{~min}$. $50 \mu \mathrm{g}$ proteins were separated using 15\% SDS-GAGE. After cell supernatants were transfered to new tubes, the protein concentration was measured. The proteins were loaded to gel prior to electrophoresis. Then, the protein was transferred to Nylon membrane. Before the membrane was probed with primary antibodies, the membrane was blocked with $5 \%$ nonfat dried milk. Antibody for Bax, Bcl- 2 and caspase- 3 were purchased from Abcam. The relative protein expression was detected using Image-Pro Plus software, version 6.0 (Media Cybernetics, Inc., Rockville, MD, USA). The protein expression was presented as density ratio vs $\beta$-actin. The $\beta$-actin antibody (Abcam) was used as an internal control. Levels of all proteins were normalized to respective actin control. The control condition was normalized to 1 and all other experimental conditions were compared to this. All the experiments repeated 5 times and one-way ANOVA was performed for statistical analysis.

\section{Transfection}

Before transfection, WM266-4 and A2058 cells were seeded in $25 \mathrm{~cm}$ [2] culture flasks in completed DMEM medium. $500 \mu \mathrm{l}$ of Opti-MEM Reduced Serum Medium (Thermo Fisher Scientific, Inc) was separately added to the miRNA and lipofectamine 2000 (Thermo Fisher Scientific, Inc), and they were mixed for $5 \mathrm{~min}$. Then, mix these two mixtures into a flask and incubated for $20 \mathrm{~min}$ at room temperature. The mixture was added to the cells prior to incubation for $36 \mathrm{~h}$ for further experiments.

\section{Quantification of miRNA expression}

Total RNA was extracted from WM266-4 and A2058 cells by TRIZOl_Reagent (Life Technologies, Thermo
Fisher Scientific, Inc) following the manufacturer's protocol. Expression of 16 miRNAs was quantified by PCR array assays purchased from QIAGEN. This array experimentally verified representative miRNAs which regulate various apoptosis related genes, and their inhibition or overexpression resulted in stimulate or attenuate apoptosis. In our analysis to screen differentially expressed miRNAs between the dug treated groups and negative control group, Limma package in $\mathrm{R}$ language was used. To calculate the false discovery rate of $P$ value (FDP) and conduct multi-test, the Benjamin-Hochberg' method was used. Differentially expressed miRNAs were identified with the $P$ value $<0.05$ and fold change $>1.2$ or $<0.8$. Then the qualified miRNAs were classified as up-regulated and down-regulated groups.

\section{Real time PCR detection of miR-181a}

To detect transcriptional level of candidate miRNA, $0.5 \mu \mathrm{l}$ of the product from RT reaction of miR-181a (diluted 1:2) was combined with $0.5 \mu \mathrm{l}$ of a 20X TaqMan MicroRNA Assay, which includes probe, reverse primer, forward primer, and $5 \mu \mathrm{l}$ of $2 \mathrm{X}$ TaqMan Universal PCR Master Mix to final reaction volume of $20 \mu$ l. The expression of miRNA was then determined with the standard TaqMan microRNA assays using Bio-RAD CFX96-Realtime PCR System. Protocol cycling conditions of $95{ }^{\circ} \mathrm{C}$ for $10 \mathrm{~min}$, and then, 40 cycles of denaturation at $95{ }^{\circ} \mathrm{C}$ for $15 \mathrm{~s}$ and annealing step at $60{ }^{\circ} \mathrm{C}$ for $60 \mathrm{~s}$. After normalization to U6, alteration in expression level of miR181a between different samples were calculated. The one-way ANOVA was used for statistical analysis. All the $P$ values are derived from testing the null hypothesis that $\Delta \Delta \mathrm{Ct}$ are equal to 0 . Therefore, a small $P$ value indicates that the $\Delta \Delta \mathrm{Ct}$ is significantly different from 0 , which demonstrates a significant effect. $P<0.05$ is considered as significant difference.

\section{RNA hybrid algorithms and firefly luciferase activity}

We have identified a putative miR-181a-binding site within the 3'-UTR of Bcl-2 mRNA using Target Scan and RNA hybrid algorithms. The luciferase activity was measured prior to co-transfection of cloned plasmid, with either miR-181a or negative control miRNA, into HEK cells.

\section{Statistical analyses}

All statistical analysis was performed using SPSS software 19.0 version and data were expressed as mean \pm SD. A two-tailed $P<0.05$ was considered as statistically significant. Student's unpaired $t$ test was used for determining the differences between two group comparison and for more than two group comparisons One-way ANOVA test was used. 

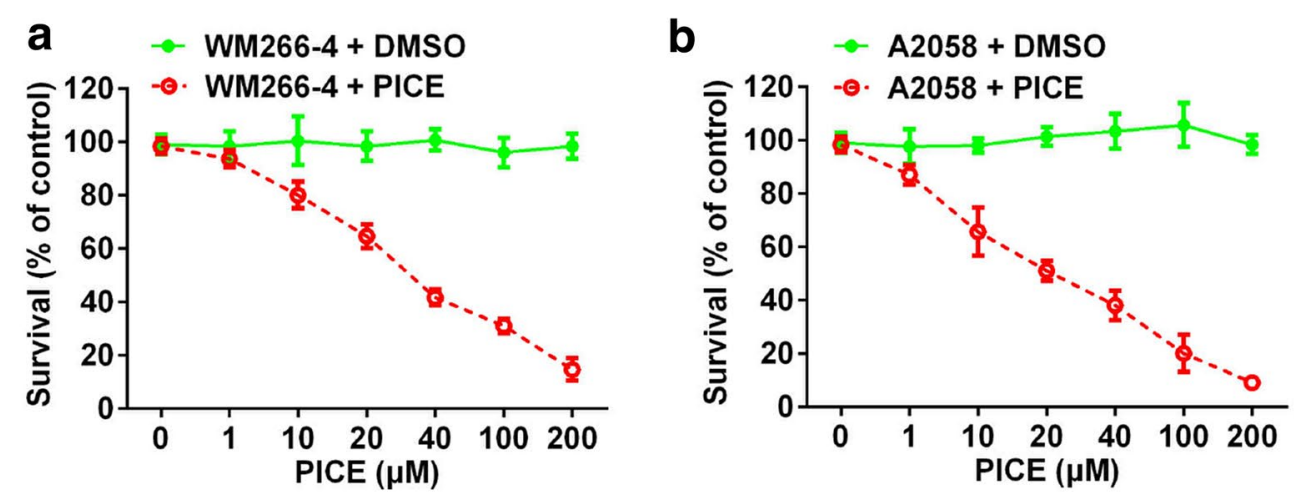

\section{C \\ WM266-4}
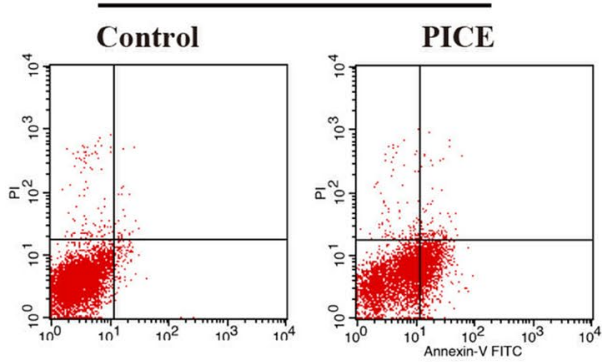

d
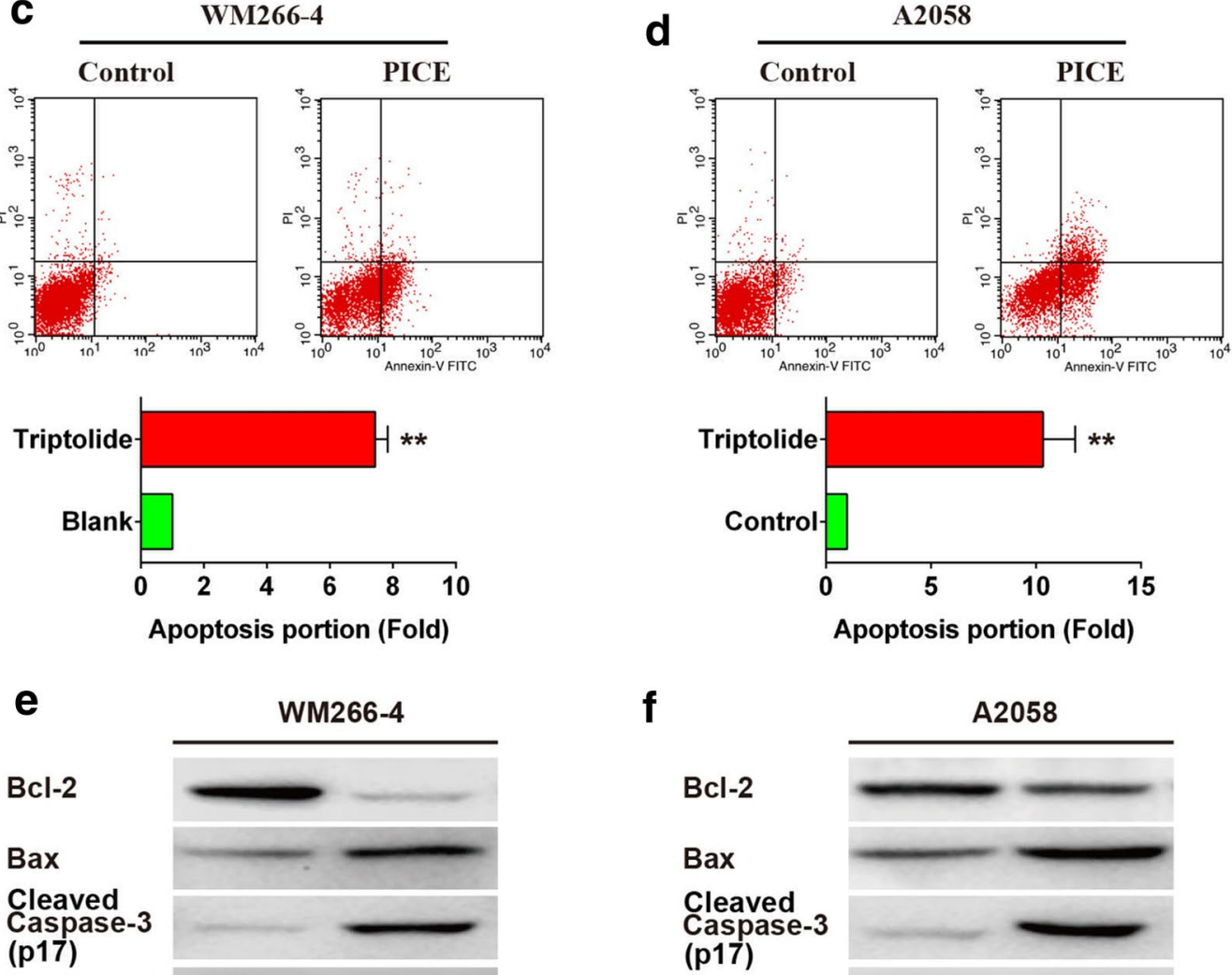

WM266-4

f
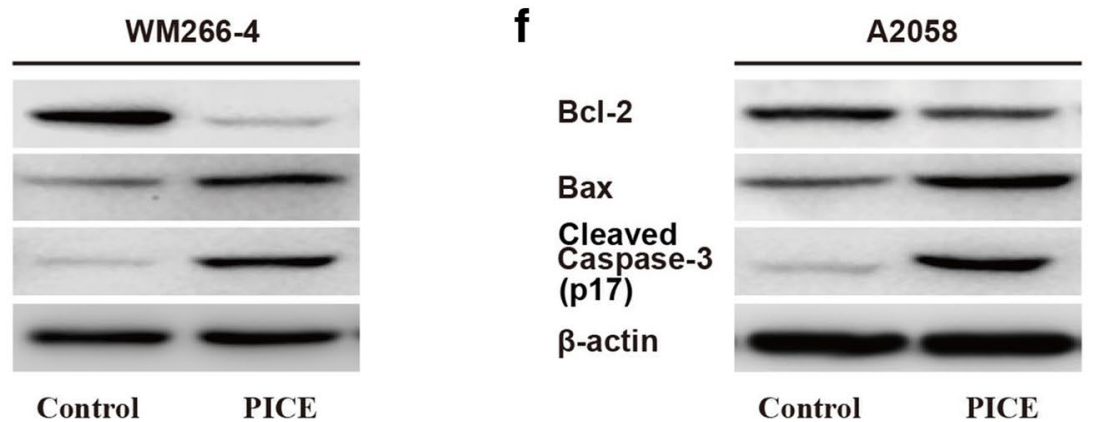

Fig. 1 Piceatannol induces apoptosis of melanoma cells. (1) Effects of different concentrations of piceatannol on cell growth of WM266-4 (a) and A2058 (b); (2) Piceatannol increases apoptosis of WM266-4 (c) or A2058 (d) cells, as measured by flow cytometry; (3) Protein level of Bcl-2, Bax and activated caspase-3 (p17) in WM266-4 (e) and A2058 (f) cells incubated with piceatannol, as demonstrated by western blot. ${ }^{*} P<0.05$, ${ }^{* *} P<0.01$ compared to blank control

\section{Results}

Piceatanal induces growth and apoptosis of melanoma cells WM266-4 and A2058

To investigate the role of piceatannol on the growth of melanoma cells, WM266-4 and A2058 cells were treated with piceatannol at different concentration for $36 \mathrm{~h}$, and then the cell activity was determined by MTT Assay. The result was shown in the Fig. 1a, the growth of WM266-4 cells was restrained with an $\mathrm{IC}_{50}$ of $29.4 \mu \mathrm{M}$ piceatannol in a dose-dependent manner whereas the growth inhibitory effect was observed with $\mathrm{IC}_{50}$ of $15.6 \mu \mathrm{M}$ piceatannol in A2058 cell line (Fig. 1b). Thus, $30 \mu \mathrm{M}$ of piceatannol concentration was used for the subsequent analysis. 
To determine whether the decrease of cell vitality was related to cell apoptosis, we detected the apoptotic action of WM266-4 and A2058 cells treated with piceatannol using flow cytometry assay. In comparison with control group, apoptotic effect in WM266-4 and A2058 cells were notably increased with dose of $30 \mu \mathrm{M}$ piceatannol treatment (Fig. 1c, d). It was needed to be further determined whether the piceatannol-induced apoptosis of melanoma cells was involved in the expression of pathway regulatory proteins, caspase- $3, \mathrm{Bcl}-2$ and Bax. For this reason, western blot was conducted to test the expression change of these three proteins and we found that expression of caspase- 3 and Bax was increased while the expression of $\mathrm{Bcl}-2$ proteins was markedly decreased in the cells treated with piceatannol compared with control group (Fig. 1e, f).

\section{Piceatannol promotes expression of miR-181a}

Compared to their respective controls, the differentially expressed miRNAs in treatment group have been identified and classified into up-regulated and down-regulated groups, in accordance with the principle that ratio should be more than 2 comparing with control. It was shown that miR-181a was remarkably upregulated providing a reason to conduct further investigation on this miRNA (Fig. 2a). To validate the screened out result by PCR array, the expression of miR-181a was quantified by real-time RT-PCR. The result showed that the level of miR-181a was increased in both WM266-4 and A2058 cells treated with piceatannol for $48 \mathrm{~h}$ (Fig. $2 \mathrm{~b}$ ). To confirm the previous result of miR-181a accurately, 10 melanoma tissues and 10 normal tissues were subjected to quantitative RTPCR for measuring the expression of miR-181a in tissues.

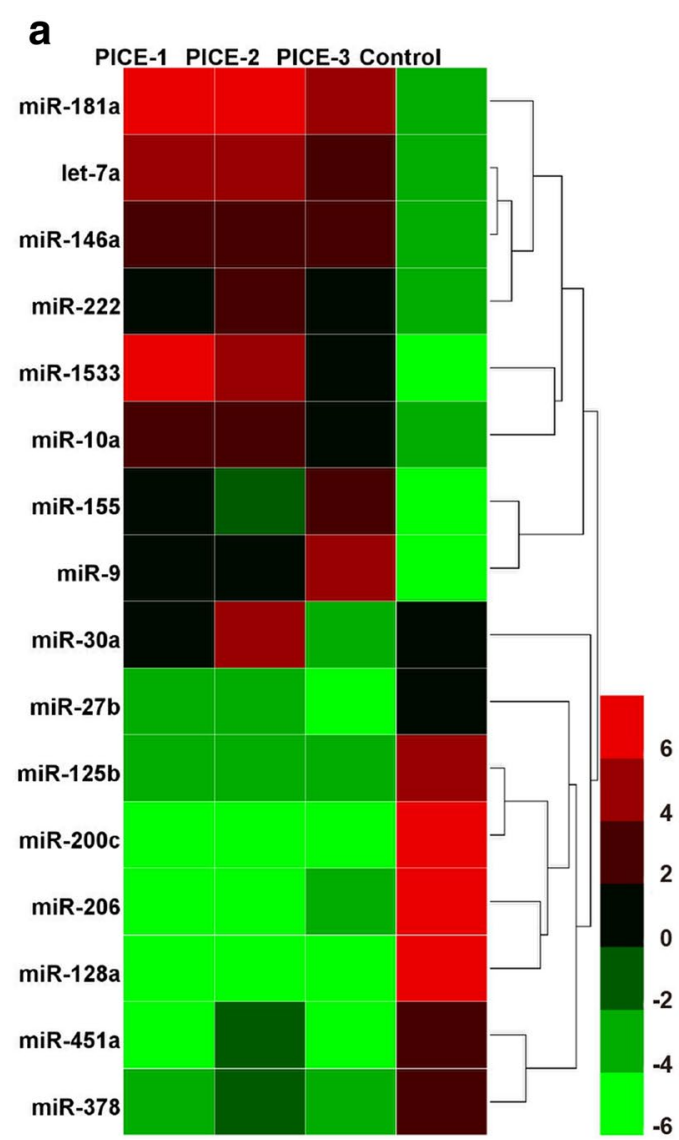

b

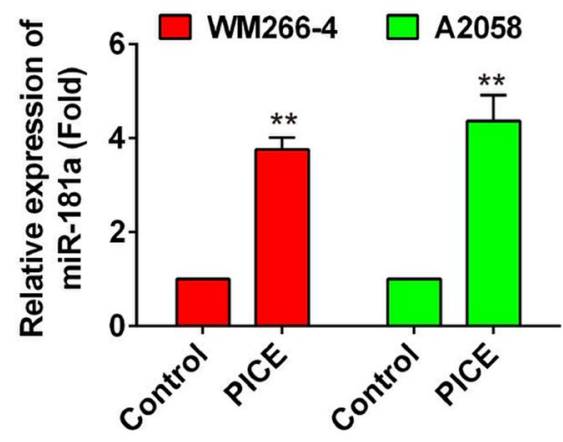

C

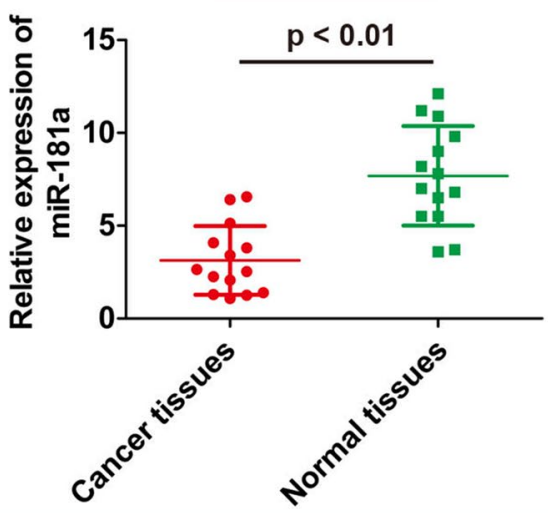

Fig. 2 Piceatannol promotes the expression of miR-181a. a Unsupervised analysis heat map of 4 different samples. All samples are underwent cell clustering, and the top 16 microRNAs with highest standard deviation (SD >1) are enlisted. Each column represents one specimen and each row represents one microRNA. The color scale from 6 to -6 shown at the right bottom of color map indicates the relative expression of a miRNA in all subjected samples: green color represents expression level lower than control while red color represents an expression level higher than control, 8 up-regulated and 8 down-regulated miRNAs were shown on the map; b Relative expression of miR-181a with and without piceatannol treatment in WM266-4 and A2058 cells; c miR-18a relative expression level in cancer tissues compared with normal tissues was analyzed using qRT-PCR. ${ }^{*} P<0.05,{ }^{* *} P<0.01$ compared to normal control 
a

miR-181a 3' ugaguggCUGUCGCAACUUACAa 5'

Bcl-2 wt 5' cauguuaGA--AGCAAUGAAUGUa 3'

Bcl-2 mut 5' cauguuaGA-AGCAACAGCACAa 3'

C

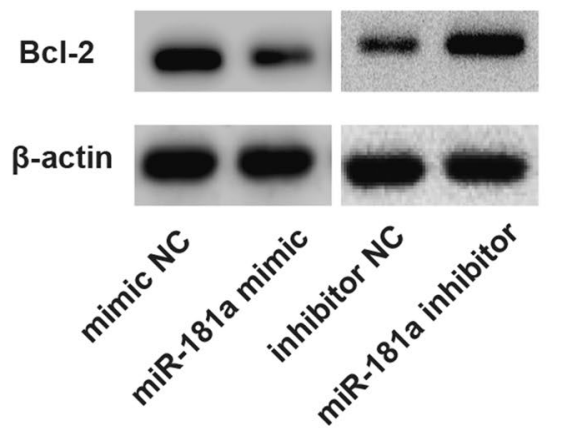

b
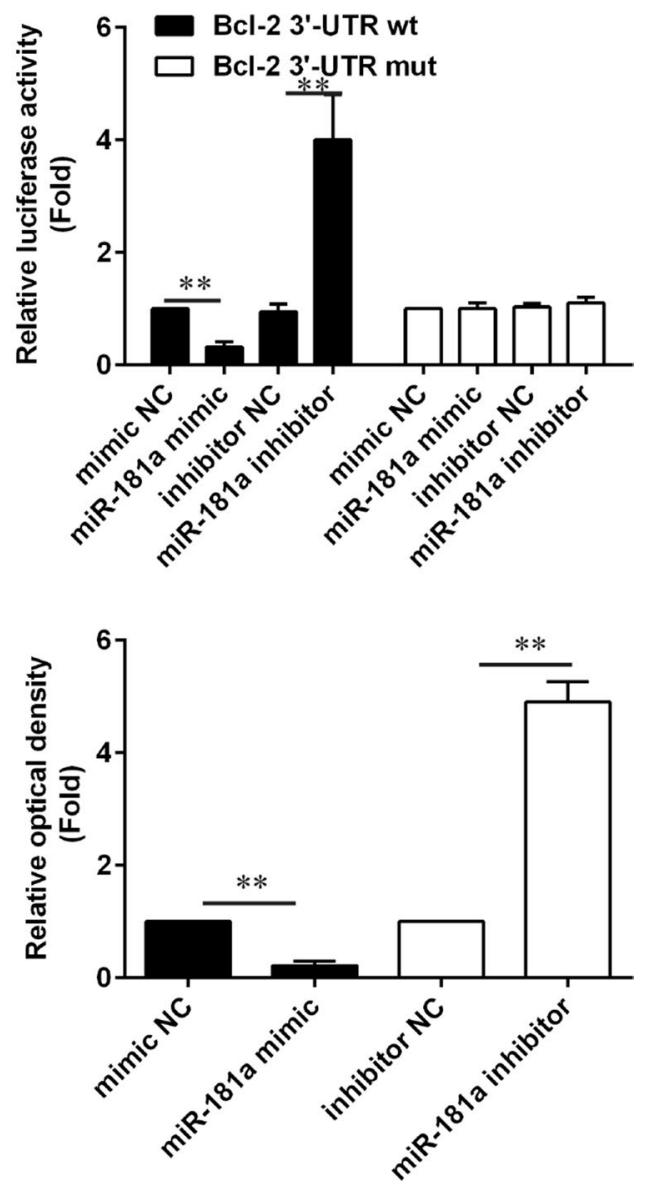

Fig. $3 \mathrm{BCl}-2$ is the direct target of miR-181a. a Schematic representation of BCl-2 mRNA with a putative 3'-UTRmiR-181a-binding site and sequences of wildtype (Bcl-2WT) and mutant (BCl-2mut) miR-181a target sites. Seed region is framed; $\mathbf{b}$ Luciferase activity of 3'-UTR-Bcl-2 (wt) was inhibited with Transfection Of miR-181a and such inhibition was not present in the miR-181a-binding site (mut) with mutations or with participation of miR181 a inhibitor. The influence of miR-181a on expression level of $\mathrm{BCl}-2$ was compared to the mimic negative control; c Western blotting analysis of $\mathrm{BCl}-2$ and $\beta$-actin protein levels after transfection of miR-181a or mimic NC, or miR-181a inhibitor or inhibitor NC. ${ }^{* *} P<0.01$

The expression of miR-181a was remarkably decreased in melanoma tissues than in normal tissues (Fig. 2c).

\section{$\mathrm{BCl}-2$ is a direct target of miR-181a in melanoma cells}

$\mathrm{Bcl}-2$ is a one of the regulatory molecules for the cell apoptosis. The putative miR-181a binding site was identified within the 3'-UTR of Bcl-2 mRNA by Target Scan analysis (Fig. 3a). To test the direct binding of miR-181a to this site, wild type or mutant full length Bcl-23'UTR luciferase reporter constructs were generated. The recombinant $\mathrm{Bcl}-23^{\prime}-\mathrm{UTR}$ constructs were transfected into HEK cells together with miR-181a mimic or miR181 inhibitor. We observed that in HEK cells transfected with Bcl-2 wild type constructs, the luciferase activity was significantly inhibited by miR-181a compared to the controls, indicating that miR-181a can directly interact with the 3'-untranslated region of Bcl-2 mRNA.
Plus, miR-181a did not restrain the luciferase activity of $\mathrm{Bcl}-2$ with mutations in the miR-181a-binding site (Fig. 3b). The western blot analysis was conducted to determine protein expression of $\mathrm{Bcl}-2$ and we uncovered that miR-181a decreases the $\mathrm{Bcl}-2$ protein in HEK cells compared to the negative control miRNA while the $\mathrm{Bcl}-2$ protein expression was increased with miR-181a inhibitor (Fig. 3c). These results indicated that miR-181a directly targets the Bcl-2 3'-UTR to suppress Bcl-2 protein translation.

\section{Silencing of miR-181a mitigates the pro-apoptotic effects of piceatannol in melanoma cells}

To determine whether the overexpression of miR-181a is participate in the piceatannol-induced apoptosis in melanoma cells, transfected specific inhibitor was used to knockdown the miR-181a, and we monitored the 

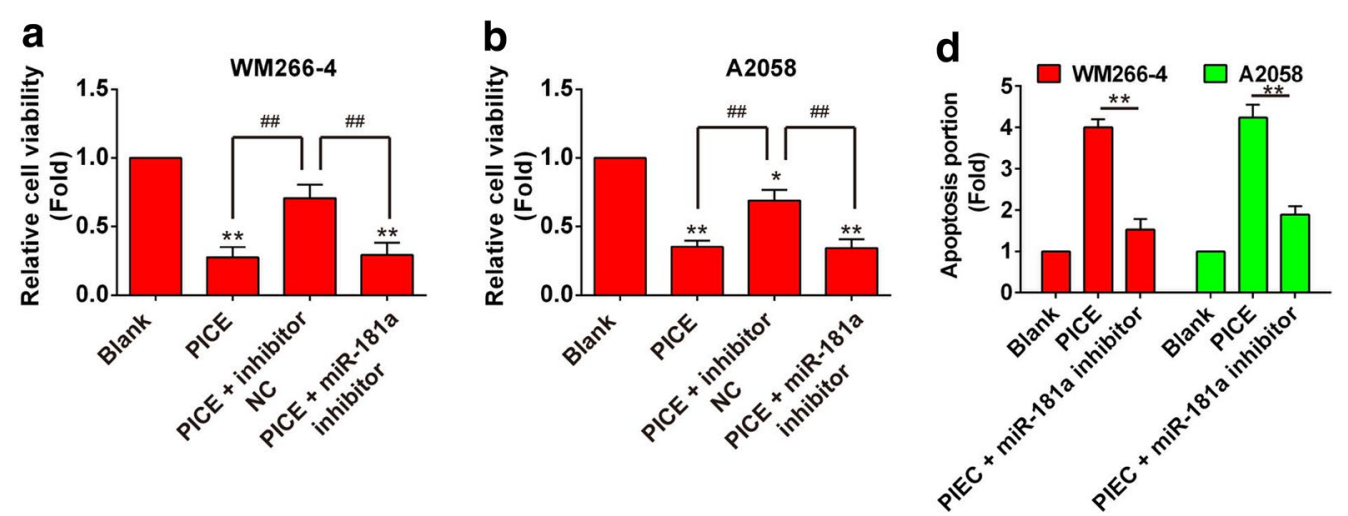

C
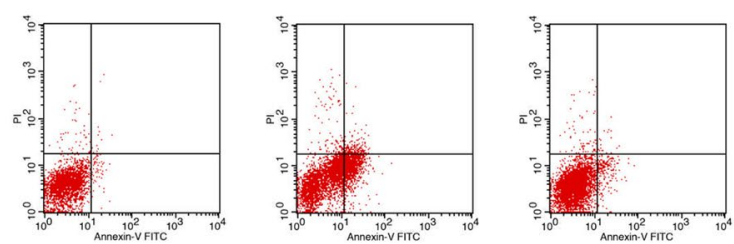

WM266-4
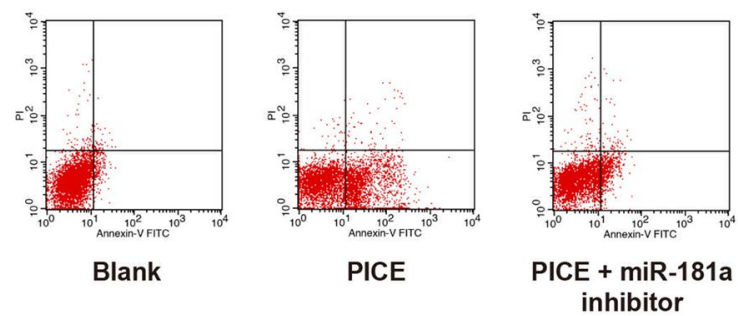

A2058
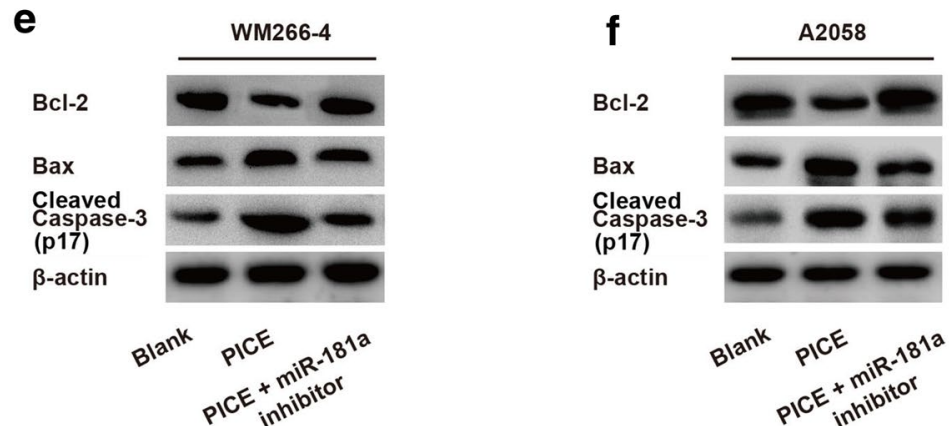

Fig. 4 miR-181a knock down reduces the apoptotic effect of piceatannol on melanoma cells. (1) Cell viability assay (MTS) of WM266-4 (a) and A2058 (b) cells transfected with miR-181a or miR-181a inhibitor after piceatannol treatment; (2) Silencing of miR-181a alleviates the fall of cell apoptotic activity induced by piceatannol in WM266-4 (c) and A2058 (d) cells; (3) Inhibition of miR-181a stimulates expression dysregulation of activated caspase-3, Bax and Bcl-2 proteins in WM266-4 (e) and A2058 (f) cells. ${ }^{*} P<0.05,{ }^{*} P<0.01$ compared to normal control, \#\#P<0.01

change of apoptotic action in melanoma cells. As shown in Fig. 4a, b, miR-181a inhibitor reinstated the reduction of cell vitality induced by piceatannol and it decreased the apoptotic role of piceatannol in melanoma WM266-4 and A2058 cells (Fig. 4c, d). The dysregulation of Bax, caspase- 3 , and $\mathrm{Bcl}-2$ caused by piceatannol was reversed by miR-181a inhibitor in WM266-4 and A2058 cells (Fig. 4e, f).

\section{Discussion}

In this study, we have demonstrated that piceatannol restrains the growth of melanoma cells. The expression of miR-181a was promoted by piceatannol and it caused up-regulation of Bax, down-regulation of Bcl-2 and activation of caspase-3, ultimately induced the apoptosis in melanoma cells. Above all, we discovered that highly expressed miR-181a induces apoptosis in melanoma 
cells, and pro-apoptotic action of piceatannol could be reduced by knockdown of miR-181a. Overall, our work demonstrated the pro-apoptotic effect of piceatannol in melanoma cells and disclosed the contribution of miR181a in this biological process. And, present study suggests that piceatannol could be a new therapeutic agent for treatment of melanoma. This study is the first to report that promotion of miR-181a induced by piceatannol offers a therapeutically beneficial option for melanoma treatment, as evidenced by using clinical samples and cell lines and analysis.

Resveratrol and its derivative piceatannol specifically cause cell death in cancer cells [20-22]. Because of their greatly enriched ER-mitochondria tethering, cancer cells are highly susceptible for resveratrol/piceatannol-induced reduction of SERCA (sarco/endoplasmic reticulum $\mathrm{Ca}^{2+}$ ATPase) activity to yield mitochondrial $\mathrm{Ca}^{2+}$ overload and subsequent cancer cell death [23]. Among trans-resveratrol analogs, such as pterostilbene, piceatannol, polydatin and oxyresveratrol, was the more promising candidate for future studies regarding treatment of leishmaniasis [24]. A number of studies showed that piceatannol participate in inhibition of biological activities, such as invasion, progression, metastasis and drug-resistance, in lung cancer cells [25], colorectal cancer [26], breast cancer [27], ovarian cancer [28], prostate cancer [29] and leukemia [30]. However, few reports have been made on the effects of piceatannol in melanoma. Piceatannol was investigated for its anti-oxidative property and ability to inhibit melanogenesis to be used as new skin lightening agent [31]. A study investigated the anti-melanoma properties of dietary piceatannol considering its potent pro-apoptotic capacity at low concentration [9]. However, potential of piceatannol as new agent in melanoma treatment has been over looked. Our results emphasize this anti-cancer role of piceatannol by exhibiting that the capability of piceatannol to restrain melanoma cells growth by partially influencing Bax, Bcl-2 and caspase- 3 expression in apoptosis, suggesting that piceatannol could be used for the treatment of melanoma.

A mRNA can be targeted by multiple miRNAs, and, conversely, a miRNA can interact with multiple mRNAs [32]. Recent experimental and clinical studies have uncovered that melanoma is influenced by various miRNAs, for instance miR-138, miR-211, miR-18b, miR339-3p, miR-542-3p and so on [33-37]. In accordance with our microarray analysis, miR-181a is distinctively overexpressed in piceatannol-treated cells compared with normal control (Fig. 2a). Therefore, we selected miR-181a as a new candidate miRNA promotes apoptosis in melanoma cells to conduct further investigation. miR-181a has previously been reported to be upregulated in diverse cancers, such as ovarian cancer [38], gastric cancer [39], leukemia [40], and be downregulated in breast cancer [41], regulating epithelial-to-mesenchymal transition, cell proliferation, migration andG1/S transition. Yet, the exact function of miR-181a in the regulation of the apoptotic effect of piceatannol in melanoma cells remains unclear and this study is first to report its regulatory function with systematic analysis. In this study, we found that miR-181a dramatically overexpressed in both WM266-4 and A2058 cells treated with piceatannol, and downregulated in melanoma cancer tissues compared with its adjacent ones. Thus we suggest that, high level of miR-181a expression might associate with apoptotic effect of piceatannol in melanoma cells.

Bcl-2 is known to be a crucial anti-apoptotic protein and miRNA mediated $\mathrm{Bcl}-2$ suppression has been demonstrated in a wide variety of cancers [42-44]. miR-181a was one of the miRNAs that downregulated in post-transcriptional level and preserve the low expression of relative proteins, such as silent mating-type information regulation 2 homologue 1 (SIRT1), which regulates various biological processes including transcription, apoptosis and muscle differentiation by deacetylating key proteins [45]. As estimated to be potent regulator in cell apoptosis by specifically targeting Bcl-2, miR-181a has been subjected to diverse level of tests in many different cancers and its functions are well documented [46]. A research presented evidence that in vivo allergic inflammation promotes the metastatic potential of mouse melanoma cells and miR-181a would be a valuable target for the development of anti-allergic drugs [47]. Several pieces of evidence suggested that the diminished expression of miR-181a in superficial spreading melanoma may contribute to the onset of melanoma subtype [48]. However, to date underlying mechanism of interaction between miR-181a and Bcl-2 in melanoma cells has not been fully understood and here we investigated into miRNA-protein interaction by defining specific binding sites in melanoma cells, and our findings would provide some insights into mechanism of anti-cancer effect of piceatannol. In accordance with the data presented in this study, it is shown that $\mathrm{Bcl}-2$ is a direct target of miR181a, via an independent binding site in its $3^{\prime}$-UTR, which reduces its expression, activity and downstream signaling, and our functional experiments validate $\mathrm{Bcl}-2$ as a critical mediator of the pro-apoptotic effects of miR-181a in melanoma cells. The knockdown results of miR-181a contribute to make a further validation of these data. However, our data do not address whether miR-181a is involved in melanoma progression and invasion.

\section{Conclusions}

To sum up, our findings showed that piceatannol promoted the expression to miR-181a, which mediated pro-apoptotic effect of piceatannol by suppressing Bcl-2 
in melanoma cells. It provides basic information to better understanding the molecular mechanism involved in the anti-cancer activity of piceatannol in melanoma cells and suggested that piceatannol could be a potent agent for melanoma treatment. To best of our knowledge, this study is the first to report that systematically investigated the anti-cancer effect of piceatannol by probing into its mediatory miRNA for melanoma cells. It is needed to conduct further investigation on validation of therapeutic functions of piceatannol for melanoma in vivo, and should be tested against melanoma in clinical practice.

\section{Abbreviations \\ CCK-8: Cell Counting Kit-8; OD: optical density; FDP: false discovery rate of P value; SIRT1: silent mating-type information regulation 2 homologue 1.}

\section{Authors' contributions}

Conception and design of entire study: MD, ZZ. Analysis and interpretation of data: MD, ZZ, TG. Drafted the article: ZZ, TG. Revised it critically for content: MD. Other: ZZ, TG. All authors read and approved the final manuscript.

\section{Author details \\ 1 Department of Dermatology, The Second Affiliated Hospital of Chongqing Medical University, 76 Linjiang Road, Yuzhong District, Chongqing 400010, China. ${ }^{2}$ Department of Dermatology, Chongqing Traditional Chinese Medi- cine Hospital, Chongqing 400021, China.}

\section{Acknowledgements}

Not applicable.

\section{Competing interests}

The authors declare that they have no competing interests.

\section{Availability of data and materials}

All data generated or analysed during this study are included in this published article.

\section{Consent for publication}

Not applicable.

\section{Ethics approval and consent to participate}

This study was approved by ethics review board of Chongqing Medical University and all the patients provided written informed consent.

\section{Funding}

The study was supported by no funding.

\section{Publisher's Note}

Springer Nature remains neutral with regard to jurisdictional claims in published maps and institutional affiliations.

Received: 12 April 2017 Accepted: 22 September 2017 Published online: 17 October 2017

\section{References}

1. Trotter SC, Sroa N, Winkelmann RR, Olencki T, Bechtel M. A global review of melanoma follow-up guidelines. J Clin Aesthetic Dermatol. 2013;6(9):18.

2. Rastrelli M, Tropea S, Rossi CR, Alaibac M. Melanoma: epidemiology, risk factors, pathogenesis, diagnosis and classification. In vivo. 2014;28(6):1005-11.

3. Group UCSW. United States cancer statistics: 1999-2012 incidence and mortality web-based report. Atlanta: Department of Health and Human
Services, Centers for Disease Control and Prevention, and National Cancer Institute; 2015.

4. Weir HK, Marrett LD, Cokkinides V, et al. Melanoma in adolescents and young adults (ages 15-39 years): United States, 1999-2006. J Am Acad Dermatol. 2011;65(5):S38.

5. Nevala WK, Buhrow SA, Knauer DJ, Reid JM, Atanasova EA, Markovic SN. Antibody targeted chemotherapy for the treatment of melanoma. Cancer Res. 2016;76:3954-64.

6. Guennoun A, Sidahmed H, Maccalli C, Seliger B, Marincola FM, Bedognetti $D$. Harnessing the immune system for the treatment of melanoma: current status and future prospects. Expert Rev Clin Immunol. 2016;12:879-93.

7. Piotrowska H, Kucinska M, Murias M. Biological activity of piceatannol: leaving the shadow of resveratrol. Mutat Res. 2012;750(1):60-82.

8. Jancinova V, Perecko T, Nosal R, Svitekova K, Drabikova K. The natural stilbenoid piceatannol decreases activity and accelerates apoptosis of human neutrophils: involvement of protein kinase C. Oxid Med Cell Longev. 2013;2013:136539.

9. Larrosa M, Tomás-Barberán FA, Espín JC. The grape and wine polyphenol piceatannol is a potent inducer of apoptosis in human SK-Mel-28 melanoma cells. Eur J Nutr. 2004;43(5):275-84.

10. Ambros $V$. The functions of animal microRNAs. Nature. 2004:431(7006):350-5.

11. Xin'en H. Action mechanisms of MicroRNAs in gastric cancer and its application in translational medicine. J Int Transl Med. 2015;3(3):217-22.

12. Garzon R, Calin GA, Croce CM. MicroRNAs in cancer. Annu Rev Med. 2009:60:167-79.

13. Bartel DP. MicroRNAs: genomics, biogenesis, mechanism, and function. Cell. 2004;116(2):281-97.

14. Rothschild SI. microRNA therapies in cancer. Mol Cell Ther. 2014;2(1):1.

15. Naidu S, Magee P, Garofalo M. MiRNA-based therapeutic intervention of cancer. J Hematol Oncol. 2015;8(1):1.

16. Bader A, Brown D, Stoudemire J, Lammers P. Developing therapeutic microRNAs for cancer. Gene Ther. 2011;18(12):1121-6.

17. Ertel F, Nguyen M, Roulston A, Shore GC. Programming cancer cells for high expression levels of Mcl1. EMBO Rep. 2013;14(4):328-36.

18. Hilmi C, Larribere L, Giuliano S, et al. IGF1 promotes resistance to apoptosis in melanoma cells through an increased expression of BCL2, BCL-X (L), and survivin. J Invest Dermatol. 2008;128(6):1499-505.

19. Hsu T, Hsu C, Lee K-H, et al. MicroRNA-18a is elevated in prostate cancer and promotes tumorigenesis through suppressing STK4 in vitro and in vivo. Oncogenesis. 2014;3(4):e99.

20. Tinhofer I, Bernhard D, Senfter M, et al. Resveratrol, a tumor-suppressive compound from grapes, induces apoptosis via a novel mitochondrial pathway controlled by Bcl-2. FASEB J. 2001;15(9):1613-5.

21. Qin $Y, M a Z$, Dang $X, L i W, M a$ Q. Effect of resveratrol on proliferation and apoptosis of human pancreatic cancer MIA PaCa-2 cells may involve inhibition of the Hedgehog signaling pathway. Mol Med Rep. 2014;10(5):2563-7.

22. Nie P, Hu W, Zhang T, Yang Y, Hou B, Zou Z. Synergistic induction of erlotinib-mediated apoptosis by resveratrol in human non-small-cell lung cancer cells by down-regulating survivin and up-regulating PUMA. Cell Physiol Biochem. 2015;35(6):2255-71.

23. Madreiter-Sokolowski CT, Gottschalk B, Parichatikanond W, et al. Resveratrol specifically kills cancer cells by a devastating increase in the $\mathrm{Ca}^{2}+$ coupling between the greatly tethered endoplasmic reticulum and mitochondria. Cell Physiol Biochem. 2016:39(4):1404-20.

24. Passos CL, Ferreira C, Soares DC, Saraiva EM. Leishmanicidal effect of synthetic trans-resveratrol analogs. PLoS ONE. 2015;10(10):e0141778.

25. Xu B, Tao Z-Z. Piceatannol enhances the antitumor efficacy of gemcitabine in human a549 non-small cell lung cancer cells. Oncol Res. 2015;22(4):213-7.

26. Zhang DZ, Lau KM, Chan ES, et al. Cell-free urinary microRNA-99a and microRNA-125b are diagnostic markers for the non-invasive screening of bladder cancer. PLOS ONE. 2014;9(7):e100793.

27. Song NR, Hwang MK, Heo Y-S, Lee KW, Lee HJ. Piceatannol suppresses the metastatic potential of MCF10A human breast epithelial cells harboring mutated $\mathrm{H}$-ras by inhibiting MMP-2 expression. Int J Mol Med. 2013;32(4):775-84.

28. Farrand L, Byun S, Kim JY, et al. Piceatannol enhances cisplatin sensitivity in ovarian cancer via modulation of p53, X-linked inhibitor 
of apoptosis protein (XIAP), and mitochondrial fission. J Biol Chem. 2013;288(33):23740-50.

29. Dias SJ, Li K, Rimando AM, et al. Trimethoxy-resveratrol and piceatannol administered orally suppress and inhibit tumor formation and growth in prostate cancer xenografts. Prostate. 2013;73(11):1135-46.

30. Liu W-H, Chang L-S. Suppression of Akt/Foxp3-mediated miR-183 expression blocks Sp1-mediated ADAM17 expression and TNFa-mediated NFKB activation in piceatannol-treated human leukemia U937 cells. Biochem Pharmacol. 2012;84(5):670-80.

31. Yokozawa T, Kim YJ. Piceatannol inhibits melanogenesis by its antioxidative actions. Biol Pharm Bull. 2007;30(11):2007-11.

32. Lim LP, Lau NC, Garrett-Engele P, et al. Microarray analysis shows that some microRNAs downregulate large numbers of target mRNAs. Nature. 2005;433(7027):769-73.

33. Chen Y, Cao K, Wang S, et al. MicroRNA-138 suppresses proliferation, invasion and glycolysis in malignant melanoma cells by targeting HIF-1a. Exp Ther Med. 2016;11(6):2513-8.

34. Yu H, Yang W. MiR-211 is epigenetically regulated by DNMT1 mediated methylation and inhibits EMT of melanoma cells by targeting RAB22A. Biochem Biophys Res Commun. 2016;476:400-5.

35. Chen Y, Zhang Z, Luo C, Chen Z, Zhou J. MicroRNA-18b inhibits the growth of malignant melanoma via inhibition of HIF-1a-mediated glycolysis. Oncol Rep. 2016;36(1):471-9.

36. Weber CE, Luo C, Hotz-Wagenblatt A, et al. miR-339-3p is a tumor suppressor in melanoma. Cancer Res. 2016;76:3562-71.

37. Rang Z, Yang G. Wang Y-W, Cui F. miR-542-3p suppresses invasion and metastasis by targeting the proto-oncogene serine/threonine protein kinase, PIM1, in melanoma. Biochem Biophys Res Commun. 2016:474(2):315-20.

38. Li L, Xu Q, Dong Y, et al. MiR-181 a upregulation is associated with epithelial-to-mesenchymal transition (EMT) and multidrug resistance (MDR) of ovarian cancer cells. Eur Rev Med Pharm Sci. 2016;20(10):2004-10.
39. Zhao J, Nie Y, Wang H, Lin Y. miR-181a suppresses autophagy and sensitizes gastric cancer cells to cisplatin. Gene. 2016;576(2):828-33.

40. Liu X, Liao W, Peng H, et al. miR-181 a promotes G1/S transition and cell proliferation in pediatric acute myeloid leukemia by targeting ATM. J Cancer Res Clin Oncol. 2016;142(1):77-87.

41. LiY, Kuscu C, Banach A, et al. miR-181a-5p inhibits cancer cell migration and angiogenesis via downregulation of matrix metalloproteinase-14. Cancer Res. 2015;75(13):2674-85.

42. Zhang Y, Huang F, Wang J, Peng L, Luo H. MiR-15b mediates liver cancer cells proliferation through targeting BCL-2. Int J Clin Exp Pathol. 2015;8(12):15677.

43. Sun C, Liu Z, Li S, et al. Down-regulation of C-Met and BCl2 by microRNA-206, activates apoptosis, and inhibits tumor cell proliferation, migration and colony formation. Oncotarget. 2015;6(28):25533.

44. Tong Z, Liu N, Lin L, Guo X, Yang D, Zhang Q. miR-125a-5p inhibits cell proliferation and induces apoptosis in colon cancer via targeting $\mathrm{BCL}$, BCL2L12 and MCL1. Biomed Pharmacother. 2015;75:129-36.

45. Saunders LR, Sharma AD, Tawney J, et al. miRNAs regulate SIRT1 expression during mouse embryonic stem cell differentiation and in adult mouse tissues. Aging (Albany NY). 2010;2(7):415-31.

46. Seoudi AM, Lashine YA, Abdelaziz Al. MicroRNA-181a-a tale of discrepancies. Expert Rev Mol Med. 2012;14:e5.

47. Eom S, Kim Y, Kim M, et al. Transglutaminase II/microRNA-218/-181a loop regulates positive feedback relationship between allergic inflammation and tumor metastasis. J Biol Chem. 2014;289(43):29483-505.

48. Poliseno L, Haimovic A, Segura MF, et al. Histology-specific microRNA alterations in melanoma. J Invest Dermatol. 2012;132(7):1860-8.

\section{Submit your next manuscript to BioMed Central and we will help you at every step:}

- We accept pre-submission inquiries

- Our selector tool helps you to find the most relevant journal

- We provide round the clock customer support

- Convenient online submission

- Thorough peer review

- Inclusion in PubMed and all major indexing services

- Maximum visibility for your research

Submit your manuscript at www.biomedcentral.com/submit
() Biomed Central 